\title{
Toward a Computational Model of the Upper Extremity
}

\author{
Hooshang Hemami ${ }^{1}$
}

\author{
${ }^{1}$ Dept. of Electrical and Computer Engineering, The Ohio State University, Columbus, Ohio 43210, USA \\ Correspondence: Hooshang Hemami, Dept. of Electrical and Computer Engineering, The Ohio State University, Colum- \\ bus, Ohio 43210, USA. E-mail: hemami.1@ osu.edu
}

Received: September 14, 2017

Accepted: September 25, 2017

Online Published: November 29, 2017

doi:10.5539/mer.v7n2p40

URL: https://doi.org/10.5539/mer.v7n2p40

\begin{abstract}
A basic 22-segment model of the upper extremity is formulated that can allow computational testing of hypotheses about the control and coordination of the upper extremity by the central nervous system. The formulation allows for further analytical, anatomical, physiological, and bio-mechanical expansion and improvement of the model. It allows for inclusion of all passive structures: ligaments, membranes, soft tissues, and cartilages. The formulation is based on the state space formulation of the Newton-Euler method applied to multi-body systems. Extensive use is made of three-segment rigid body modules, constraints, reduction of dimensionality, projection, and matrices of large dimensions.

An example, gliding motion of a rigid body on a circular surface (as in wiping a dish with a pre-specified force of contact) shows the application of some of the concepts and feasibility of the developed routines. The control is based on analogous strategies in living systems where co-activation of agonist-antagonist muscular systems and precise reference inputs implement the desirable trajectories of motion and where an integral feedback of the force implements the desired forces of contact.
\end{abstract}

Keywords: upper extremity, state space representations, computational dynamics, joints models, projection, coordination

\section{Introduction}

The problem considered here is one step in the direction of developing a computational model of the upper extremity for the following long range purposes:

1) allow computational and neural structures of the central nervous system (CNS) to be designed, tested and improved,

2) allow computational experiments that cause fracture, damage, and injury to passive and active structures of the human body; and 3) help the design of artificial limbs and their interface to the natural system.

The model consists of four rigid body segments representing two shoulder segments and the upper and lower arm, plus eighteen segments, three for each finger and three for the palm (see Boileau Grant, 1962, frames 1 and 2). The joints are all three-degree-of-freedom point connections as discussed in volume 1 of Kapandji (1979). The muscles are all single joint, allow co-activation Basmajian (1978) and are all paired as agonist-antagonist (Henneman, 1980; Warfel, 1978; Rosse \& Clawson, 1970). With such a model, numerical algorithms and software packages can be used for simple testing of conjectures and hypotheses regarding the sense of touch and functioning of the CNS (R. Kandel E. \& Jessell 1991; Kornhuber, 1971, 1973). In addition, the development here allows testing and implementation of control and postural stability apparatus in mechanical and humanoid robots. One can study daily voluntary human sport activities and maneuvers and postural strategies (Nashner \& McCollum, 1985; Brooks, 1986; Pai \& Iqbal, 1999). More detail about the skeletal structure of the model can be found in Hemami et al. (2016) and Hemami \& Hemami (2016).

We introduce a simple strategy of coordinated control that is primarily involved with the actuator forces and implements piecewise linear trajectories of force. There is a feed-forward component of force that controls the stable equilibrium points of the rigid body Winter et al. (1998). The transition of the rigid body from one equilibrium point to another is brought about by linear interpolation of the feed-forward component between the two equilibrium points. The feedback component of the control involves linear position and velocity feedback, i.e., a unity gain state feedback system with high gain in the feed-forward loop Ozbay (2000) and Byrnes \& Issidori (1991).

This strategy of control (Evarts et al., 1971; Evarts, 1975; E. Kandel et al., 1991) is consistent with a number of hypotheses (Doeringer \& Hogan, 1998; Iqbal \& Roy, 2009) and experiments Kornhuber (1973) about the CNS being involved in the production of "ramp-type" signals generated in the basal ganglia and the reticular formation that propagate to the spinal cord. It is also consistent with other findings that long loop reflexes may not be involved in postural adjustment 
maneuvers (Szturm \& Fallang, 1998).

The model does not handle operation of passive structures like ligaments, cartilages, soft tissue, and membranes; however, it may be adequate for the development of CNS strategies, structures, and neural processing.

For example, the control of support forces involves sensing these forces, introducing desired support forces, implementing integrators (with respect to time), and distributing the required load among actuators. Evidence for the existence of such a feedback loop is surmised from several sources. Henneman (1980) has discussed existence of cutaneous reflexes in the spinal cord and their role in withdrawal from potentially harmful injury. Kuffler and Nicholls (W. \& Nicholls, n.d.; Nicholls et al., 1992) have discussed existence of amplifiers (temporal facilitators) and integrators (temporal summation) in lower forms of life. More recently Prescott and De Koninck Prescott \& Kononck (2005) have shown how inward currents of sodium and calcium change properties of neurons such that they behave more like time integrators. When the injury to the spinal cord is severe, and if the functions of force control are interrupted, the control of movement that is implemented may not be adequate for support force control (Lacquaniti \& Maioli, 1994; Ivanenko et al., 2002).

The philosophy of control is to use state and integral force of constraint feedback for all constrained movements. The reference or so called "central" inputs differ for different motions. The control is partially motivated by natural systems. State feedback is available through the structure of the actuators. Co-activation and high gain implement the control. Contact force feedback is available through sensors. The inputs, at all times, are the desired trajectories of the state, and the desired force of contact trajectories. The reference state trajectories satisfy the required coordination constraints, and are centrally available. Finally, these reference trajectories are piecewise linear functions of time as stated before. The model allows testing other classes of inputs that could be trajectories and contact force candidate outputs of the CNS.

The system is assumed to be fully actuated, i.e., for every degree of freedom of the rigid body there is a control input. This means an input vector applies moment of force (torque) in the directions of roll, pitch and yaw respectively. Another force vector applies forces along the axes of roll, pitch and yaw. A force acting on the rigid body along the gradient to the surface of constraint is a third control input for controlling the force of contact.

Formulation of the upper extremity dynamics is briefly discussed in Section Two. Models of the involved natural joints are discussed in Section Three. The implementation of the constrains is presented in Section Four. A simple example of coordinated movement, demonstrating the feasibility of the models is presented in Section Five, and discussions and conclusions follow in Section Six.

\section{Formulation of the Upper Extremity Dynamics}

The objective here is to lay the foundation for deriving the equations of motion of a skeletal model for the upper extremity of humans. The simplified model considered here is composed of 22 rigid bodies representing the two shoulder girdle elements, arm and forearm elements, and six three-segment components (the palm and the five fingers.) Two different models can be envisioned: healthy movements and situations where no injuries, accidents and dislocations take place; and environments in which the system is subject to large stresses and strains where the passive structures may break, mal-function, etc. Both models allow for inclusion of all muscles and also sub-muscles when a muscle provides forces in different directions at different times. For ease of presentation, the system with passive structures included is presented first.

\subsection{With Passive Structures}

The model here is composed of 22 rigid bodies, each with three degrees of translation and three degrees of rotation. Therefore, the system has 132 degrees of freedom. All muscles, ligaments, membranes and soft tissues that connect the 22 segments must be modeled as force generators Their points of origin and insertion must be specified Hemami \& Hemami (2014). In humans, a number of upper extremity muscles function and provide force in different directions. An accurate computational model requires development of sub-muscles for such muscles for which known points of insertion and origin can be assigned. This issue is not addressed in the present paper.

\subsection{No Passive Structures}

We assume there are no injuries, accidents, dislocations, i.e.,so the joints remain healthy and function normally (Kendall et al., 1971; Tomovic et al., 1995). Every one of the 22 segments has three degrees of freedom and the torso is assumed to be stationery and correspond to the the inertial coordinate system (ICS) to which the upper extremity is joined. The formulation of the skeletal system starts with writing the 12 Newton-Euler state space equations for each of the 22 segments and, by projection, eliminating all the translational position and velocity dimensions. One may keep the system with 66 degrees of freedom. A preferred system would be to further reduce the degrees of freedom due to joint constraints of the upper extremity. The sterno-clavicular (SC), acrimo-clavicular (AC) and the glenohumeral (GH) joints, each have three degrees of freedom. The humeroulnar (HU) and the radio-ulnar (RU) joints each have two degrees of freedom. The 
finger joints have one degree of freedom and the finger-to-palm joints each have two degrees of freedom. The further reduction of the system dimensionality, due to joint constraints, without introducing natural bio-mechanical structures is considered briefly later in the section on joints (Quental et al., 2012; Kapandji, 1979).

To keep the computations, programming and notation simple, it is very important to use a minimal number of coordinate systems. This restriction helps the development of good, efficient and reusable software. Only two coordinate systems are introduced and used here. The ICS is the stationary system of the space in which the upper extremity moves. Its three right-hand axes are $\mathrm{x}$ : to the front, $\mathrm{y}$ : to the left and $\mathrm{z}$ : upwards. Every segment has its own body coordinate system, directed along its principal inertial axes. Assume the upper extremity is in its natural relaxed state. The rigid body axes are a set of convenient orthonormal right-hand axes. The $\mathrm{x}$-axis defines the front, the $\mathrm{y}$-axis the left side, and the $\mathrm{z}$-axis the vertical upwards. The motions about the closest axis to the spatial $\mathrm{x}$ axis is the body $\mathrm{x}$ axis. Similarly, the closer axis to the spatial $y$ axis is the body $y$ axis and the closer body axis to the spatial $\mathrm{z}$ axis is the body $\mathrm{z}$ axis. Three Bryant angles of roll, pitch, and yaw uniquely describe the position of every segment relative to the inertial coordinate system Paul (1982). The mechanism for this transformation is a $3 \times 3$ matrix $A$ for every one of the 22 segments, allowing transformation of a vector from the segment coordinate system to the ICS.

The position, orientation and motion of every segment is uniquely and unequivocally defined by the three Bryant angles of that segment at any and all times Shabana (1985). Failure to identify the three angles and their evolution in time creates confusion, inaccuracy and paradoxes. The Codman's paradox Kapandji (1979) is worth noting here. Abduction of the arm by 180 degrees, followed by a flexion of the arm by 180 degrees, does not bring the arm back to its original position! An additional yaw rotation of 180 degrees is also necessary to bring the arm to its original position. Precisely stated, we know that $\Theta$ is the sequence of the roll, pitch and yaw angles and $A(-\pi,-\pi, 0)$ is not the identity matrix. On the other hand,

$$
A(-\pi,-\pi,-\pi)=1
$$

To appreciate this issue, suppose an airplane performs a roll of 180 degrees followed by a pitch of 180 degrees. The airplane ends up going backward. An additional yaw of 180 degrees is needed for the airplane to resume its original orientation.

Other examples of the use of the upper extremity in circular repetitive motions that have to be specified by the time evolution of their respective Bryant angles can be cited. The Bull Roarer - invented by the Hopi for communication and other purposes - is one case. Sling mechanisms for hurling objects is another example. A similar device is used in other parts of the world to speed up the process of developing burning charcoal. The development of the dynamics is by use of state space formulation of Newton-Euler dynamics. Theoretically, one can connect together a large number of rigid bodies. The states are the Bryant angles $\Theta$ for all 22 segments and the angular velocity of every segment $\Omega$ expressed in the segment's body coordinate system.

A constraint can occur when a point of the system touches a point in the environment by design or by accident. One may touch the table with a finger. The system may touch itself intentionally or by accident, as when a new born accidentally reaches its mouth or lips with a finger and makes a point contact. Suppose $\Phi$ is the state vector of all $\Theta^{\prime} s$ involved in a given contact. Theoretically all the 66 Bryant angles could be involved in some constraint- although the involvement of each could be different. For example, the five fingers could simultaneously touch a moving object. The contact can be represented as a constraint

$$
C(\Phi)=0
$$

where $C$ is a vector that describes the contact. A permanent point of connection between two segments is a three-vector of constraints.

\section{Joints of the Upper extremity}

The human body has many joints with a variety of structures and degrees of freedom. Several such models, useful for computation and development of software, are formulated here. More realistic biomechanical joint models at the shoulder girdle are discussed by Quental et al. (2012).

\subsection{Zero Degrees of Freedom}

In the human body, there are bones that do not articulate relative to the bones next to them. Zero degrees of freedom means that two rigid bodies cannot move relative to each other. Certain natural joints require formulation of such situations.

Let three points of the rigid body be defined in the BCS system by three linearly independent vectors $R_{1}, R_{2}$ and $R_{3}$. Let the same three vectors be $D_{1}, D_{2}$, and $D_{3}$ in the ICS system. Let $R$ be a matrix whose columns are the above three vectors, and let the position and velocity of the above three points be measurable in the ICS system. Suppose the position and 
velocity of the center of gravity are also known in the ICS system. It follows that, for all $R$

$$
D=X+A R
$$

It can be shown that matrix $A$ and consequently the Bryant angles can be derived from

$$
\begin{array}{r}
A=\left[R_{1}, R_{2}, R_{3}\right]^{-1}\left[D_{1}-X, D_{2}-X, D_{3}-X\right] \\
\dot{A}=\left[R_{1}, R_{2}, R_{3}\right]^{-1}\left[\dot{D_{1}}-\dot{X}, \dot{D_{2}}-\dot{X}, \dot{D_{3}}-\dot{X}\right] \\
\dot{A}=A \breve{\Omega}
\end{array}
$$

Let the columns of $A$ be, respectively, vectors $a_{1}, a_{2}$, and $a_{3}$. From matrix $A$, the Bryant angles can be computed Waldron \& Schmiedeler (2008); Craig (1989):

$$
\begin{array}{r}
\theta_{1}=\operatorname{atan} 2\left(-\left(a_{3}(2)\right) / \cos \left(\theta_{2}\right),\left(a_{3}(3)\right) / \cos \left(\theta_{2}\right)\right) \\
\left.\left.\theta_{2}=\operatorname{atan} 2\left(a_{3}(1),\left(\left(a_{2}(1) / \cos \left(\theta_{2}\right)\right)^{2}+\left(a_{1}(1) / \cos \left(\theta_{2}\right)\right)^{2}\right)^{(} 0.5\right)\right)\right) \\
\theta_{3}=\operatorname{atan} 2\left(-a_{2}(1) / \cos \left(\theta_{2}\right), a_{1}(1) / \cos \left(\theta_{2}\right)\right)
\end{array}
$$

From the last row in Eq. 2, the angular velocity $\Omega$ can also be computed.

\subsection{Three Degrees of Freedom Joint}

The human pelvic and shoulder joints are three-dimensional joints. These joints can be abstractly modeled as making permanent contact between two rigid bodies at one point. The second body can only rotate about the first body by its three Bryant angles.

\subsection{One- and Two-Degrees of Freedom Joints}

- In general, a two-degree-of-freedom joint can be constructed from a tandem connection of two one-degree-of-freedom joints. This approach requires an additional auxiliary piece of mass, which should be integrated in the Newton-Euler equations of motion. In contrast, the one-degree-of-freedom joint is easier to imagine in several forms. Therefore, a one-degree-of-freedom joint is presented first.

Let two rigid bodies be connected at two separate physical points. The two points define a line and one body can rotate about the line relative to the second body. The rotation angle between the two bodies is the single degree of freedom between the bodies.

Two constraints Hemami \& Utkin (2002) restrict the motion between the two bodies. Consider two rigid bodies with their centers of gravity (COG) specified in the ICS at vectors $X_{a}$ and $X_{b}$. There are two points of contact between the two bodies. Point one has coordinates $P_{1}$ in the BCS of body 1 and $P_{2}$ in the BCS of body 2 . Similarly, point 2 has coordinates $R_{1}$ in the BCS of body 1 and $R_{2}$ in the BCS of body 2 . Let

$$
P_{1}-R_{1}=F_{1}
$$

Let $\Theta_{a}$ and $\Theta_{b}$ be, respectively, the Bryant angle vectors for bodies a and $\mathrm{b}$. Therefore

$$
\begin{array}{r}
\left.X_{a}+A\left(\Theta_{a}\right)\left(P_{1}\right)=X_{b}+A\left(\Theta_{b}\right) P_{2}\right) \\
\left.X_{b}+A\left(\Theta_{1}\right)\left(R_{1}\right)=X_{2}+A(\Theta-2) R_{2}\right)
\end{array}
$$

Subtracting the two equations above, and performing an inverse, one obtains the following equation

$$
F_{1}=A^{T}\left(\Theta_{a}\right) A\left(\Theta_{b}\right)\left(P_{2}-R_{2}\right)
$$

Suppose $E_{1}$ and $E_{2}$ are two independent vectors that are orthogonal to $\left(P_{1}-R_{1}\right)$. The inner product of these two vectors with Eq. 7 define the two holonomic constraints of the one-degree-of-freedom joint. If both constraints are implemented, as shown later, the joint is of degree 1. If one or the other constraint is implemented, the joint is of two degrees of freedom. There are many choices here due to the arbitrariness of vectors $E_{1}$ and $E_{2}$ and which of the two constraints is ignored. Also, theoretically, these one- and two-joint constraints are functions of all the Bryant angles of the two segments. 
As stated before, in the 22-segment model of the upper extremity, the following have three degrees of freedom: the sternoclavicular, the acromio-clavicular and the gleno-humeral. The elbow, the wrist, and all the finger joints at the palm of the hand are two-degree-of-freedom joints. The humero-ulnar and radio-ulnar are one-dimensional sub-joints of the elbow joint. All finger digit joints are one-degree-of -freedom joints.

\section{Implementation of the Constraints}

We consider two approaches to implement constraints in the dynamics: soft constraints and hard constraints. A soft constraint is a contact by soft tissue. One can push on the soft tissue or grab and pull it up. Hard constraints are often maintained by ligaments and cartilages or by contact with hard surfaces. For the soft constraint, we can include a linear spring-dashpot structure or, alternatively, position and velocity feedback. Let $C$ be the displacement of the soft tissue and $\dot{C}$, the velocity of motion of the soft tissue. Let $B_{\phi}$ be a square matrix whose diagonal elements are the sequence of $B$ matrices for the segment comprising $\Phi$. One model for the feedback contact force causing the moment of force $N_{c}$ (in three dimension) is

$$
N_{c}=B_{\phi}(\partial(C) / \partial(\Phi))\left(\left(K_{c}\right) C+\left(L_{c}\right)(\dot{C})\right)
$$

For the hard constraint case, one can show that the force of constraint is, in general, a specific function of the states and inputs (neural or muscular). With the above structure in mind, let us consider the design of one- or two-degrees-offreedom joint. The holonomic constraints that describe the resulting constraints are, as shown above, functions of the Bryant angles $\Theta_{a}$ and $\Theta_{b}$ of the two rigid bodies, the coordinates of the two points of contact on each rigid body (in their $\mathrm{BCS}$ ), and the selection of the two orthogonal vectors:

- Bryant angles $\Theta_{a}$

- Bryant angles $\Theta_{b}$

- Coordinates of the two contact points in the first BCS and the second BCS

- Orthogonal vectors to the line of contact of the two points.

With all these parameters, the design of models of elbow and wrist joints becomes challenging and time consuming. Certain simplifications may apply, and simplify the design:

- Small angles can be set to zero.

- The A matrix or its sub-components can be linearized.

- The two points on each rigid body can be selected along the principal axes of the rigid body.

- The needed orthogonal vectors can be selected along other principal axes of the rigid body.

\section{Example}

A simple example is described here to demonstrate the feasibility of the approach and illustrate the computational methodology. More detail about the kinematics, dynamics, and control of a rigid body moving on a surface can be found in Hemami \& Utkin (2002). A single rigid body, such as a kitchen wiper, is in contact with a smooth frictionless surface of a second rigid body, such as a pot. A circular frictionless pot, in the shape of a paraboloid, is the surface of constraint on which the wiper moves. The equation of the surface in ICS, is given by

$$
4 z-(x)^{2}-(y)^{2}=0
$$

There are three control inputs: vector $U_{1}, U_{2}$, and $U_{3}$. The system is assumed to be fully actuated, i.e., for every degree of freedom of the rigid body there is a control input. This means both torque vector $U_{1}$ and force vector $U_{2}$ are of dimension three. We assume also that, for controlling $\Gamma$, there is a control input: a force vector $U_{3}$ acting on the rigid body along the gradient to the surface of constraint. We further assume this force operates at the center of gravity of the rigid body, so that it is only active on the translational equations. This force input only enters the translational Eqs. of motion. For sharpening focus on the results, the gravity force is set to zero. Because we assume there is whole state feedback, a nonzero gravity vector can be easily compensated for. 
Assume the pot is on a table and is fixed to the ICS. The vertical inertial axis is $z$. The gravity vector is in the negative $z$ direction. The physical parameters of the wiper are given in the Appendix. The attitude (Bryant angles) of the wiper depend on the position of the pot along the translational trajectory, i.e., full coordination between translation and rotation.

Assume the pot is on a table and is fixed to the ICS. The vertical inertial axis is $z$. The gravity vector is in the negative $z$ direction. The wiper is supposed to follow a circular path of $\pi / 2$ degrees on the inside of the pot, remain on the surface during the motion, remain orthogonal to the surface, and always face the vertical symmetry axis of the bowl, i.e., the gravity axis. This means the wiper rotates about the vertical axis of the bowl. The wiper is assumed to have the following BCS axes like humans: front, left, and upward. They corresponding to airplane roll, pitch and yaw axes. The principal $\mathrm{z}$-axis of the wiper remains orthogonal to the surface of the pot , and the front axis of the wiper intersecting the $\mathrm{z}$-axis of the inertial coordinate system. Thus the wiper always looks at the inertial z-axis of the pot. As a consequence, the three unit vectors along the wiper body axes can be computed. The unit vector, $n_{3}$ along the z-axis of the wiper is in parallel with gradient vector to the surface. The unit vector along the wiper $y$-axis, $n_{2}$, is orthogonal to the gradient and the inertial $\mathrm{z}$-axis. The unit vector along the $\mathrm{x}$-axis, $n_{1}$, is orthonormal to the other two unit vectors. For convenience we define the $3 \times 3$ matrix $N=\left[n_{1}, n_{2}, n_{3}\right]$. Matrix $A(\Theta)$ can be derived from its definition:

$$
\left[n_{1}, n_{2}, n_{3}\right]=A
$$

Eq. 9 defines the full coordination between the rotational and the translational motion. From matrix $A$, the Bryant angles can be computed from Eq. 4. Therefore, the three translation and the three rotation motions of the wiper are defined as functions of time. These six reference inputs are centrally generated and fed forward to the system.

The initial position states of the wiper are assumed to be at

$$
\Theta_{i}=\left[\begin{array}{lll}
0, \pi / 4, & 0
\end{array}\right]^{\prime},
$$

and

$$
X=[-2,0,1]
$$

The final position is one quarter of the circular path on the pot, and remaining at the same elevation:

$$
\Theta_{f}=\left[\begin{array}{lll}
-\pi / 4, & 0, \pi / 2
\end{array}\right]^{\prime},
$$

and

$$
X_{f}=[0,-2,1]^{\prime} .
$$

The pot-wiper system is originally at equilibrium at the initial position. The motion is to start at $\mathrm{t}=0$. The six input reference trajectories are derived from the fact that the desired maneuver can be described by a simple rotation. The six central input states (rotational and translational) are shown in Fig. 1. The bias constant input $U_{3}$ signals are linearly interpolated between the initial and final positions as shown in Fig. 2. A desired constant force of constraint $\Gamma_{d}$ of 410 Newton is desired based on which the initial and final constant third bias input $U_{3}$ is computed. The duration of the motion is 2.5 seconds. The actual signals reach their terminal desired value in two seconds and the inputs are held at their final values for half a second to show the final transients. The gains of the feedback control part of the system are constant throughout the motion and are listed in the Appendix.

The results of the simulation are shown in the following four figures. The rotational and translational position outputs are shown in Fig. 3 as functions of time. The six trajectories of the translational states are shown in Fig. 4. In spite of the relatively large errors, the system reaches the final desirable state reasonably well. The desired and actual final rotational position states are:

$$
\begin{gathered}
\Theta_{r}=\left[\begin{array}{lll}
-0.7854, & 0, & 1.5708
\end{array}\right]^{\prime}, \\
\Theta=\left[\begin{array}{lll}
-0.7642, & 0.0447, & 1.5584
\end{array}\right] .
\end{gathered}
$$

The desired and actual final translational position states are:

$$
X_{r}=[0.0,-2.0,1.0],
$$




$$
X=[-0.0047,-1.9991,0.9979] .
$$

The controlled force of constraint $\Gamma$ and the condition of the constraint

$$
C=0
$$

are shown in Fig. 5. The error in the force is less than $10 \%$ and the constraint remains within four millimetes instead of zero.

The three controls $\left(U_{1}, U_{2}\right.$ and linearly interpolated bias inputs $\left.U_{3}\right)$ are shown in Fig. 6 as functions of time.

\section{Discussion and Conclusions}

A 22-segment computational model is considered for the upper extremity that does not include passive structures and all joints are of the three-dimensional single-point-of-contact type. The connection constraints allow a 66 -dimensional formulation of the dynamics with a stationary torso or a 69-degree-of-freedom system when the torso is also in motion The model integrates coordination requirements and conventional reduction of dimensionality methods. Further, the control of trajectories of motion and the forces of constraint can be relegated to two separate loops: state feedback for trajectory control and integral control for force feedback control.

The dynamics were formulated in terms of the Bryant angles, angular velocity vector, and the position and velocity of one contact point. The formulation, based on Newton-Euler state space method, is concise and yields to easy computer implementation and a simple control strategy. An example of gliding a wiper on a circular pot was formulated under the conditions of full actuation. Unity state feedback, high gains, and integral control of the force of constraint were sufficient for coordinated control of the motion. The philosophy of this control is based on what may be involved in natural systems with co-activation of muscles and use of continuous force feedback throughout the duration of the movement. The methodology may have applications in humanoid robot control and, more importantly, in neural and muscular electrical stimulation. Here we arbitrarily distributed the requirements for force control along all the actuators. In natural systems, selective muscles or muscle fibers may be involved, and the mechanisms of such selection and arbitration, not known yet, must be explored.

The human hand is involved in a very large number of diverse activities. The computational model allows experiments with potential models of both the motor and sensory components of CNS processing, structures and couplings. A few are cited here.

- Tactile sensory apparatus

- Tactile object exploration

- Recognition and identification

- Component and dimensions of tactile communication

- Role of self exploration in infant mental development and awareness

- Tactile communication with other species and animals

- Communication by hand signals

- CNS structures in all artistic endeavors

Research endeavors in these areas require development of easily accessible universal computational tools and software. They define research areas of mutual interest for all mankind. Such efforts, in addition to scientific merit, should facilitate sharing, collaboration, understanding, and giving among the people of the world. 

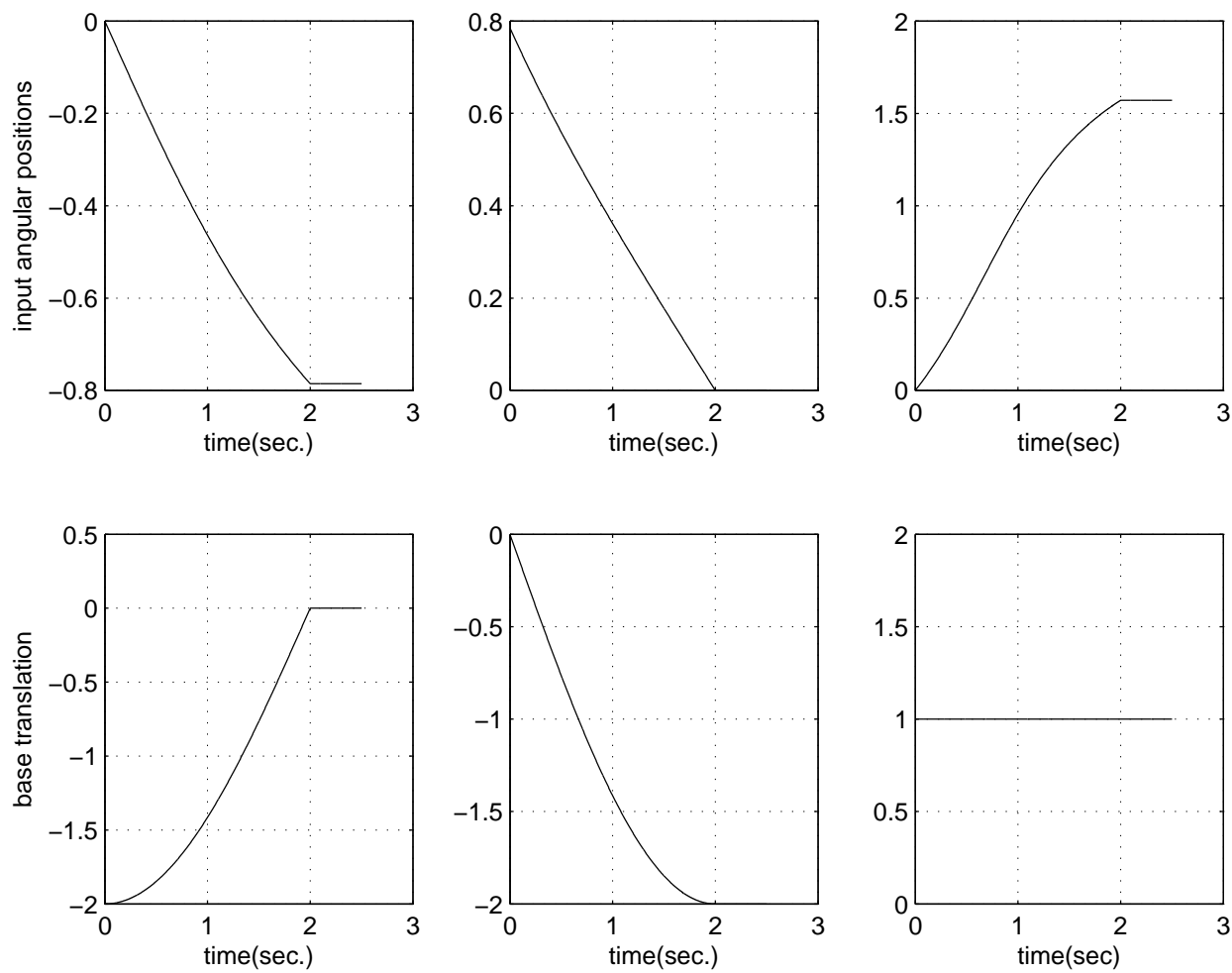

Figure 1. The reference input position state trajectories. Bryant angles are in radians and Translation states are in meters
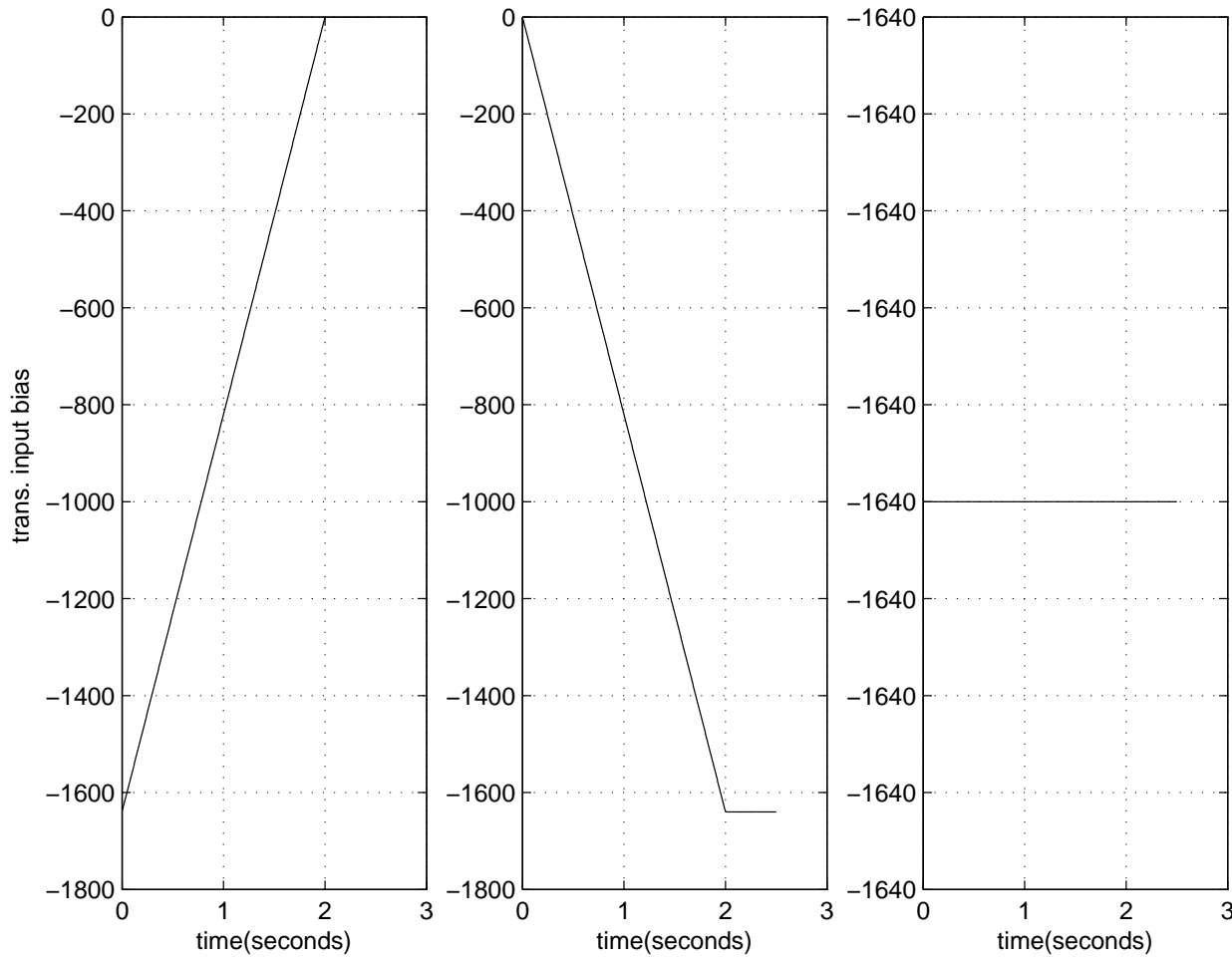

Figure 2. The interpolated bias force vector $U_{3}$ - in Newtons 

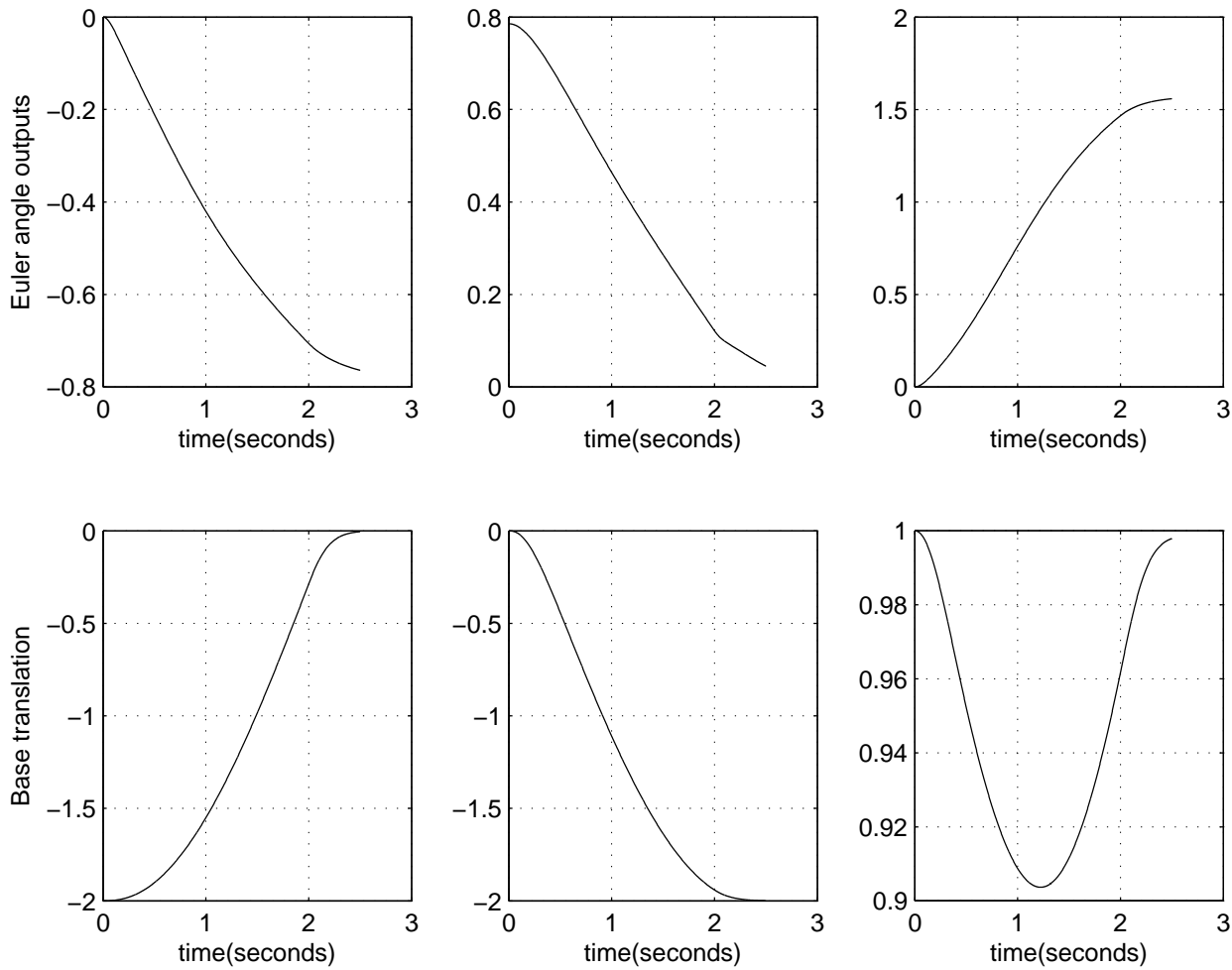

Figure 3. The position output states as functions of time The Bryant angles in radians, and the Translation states in meters.
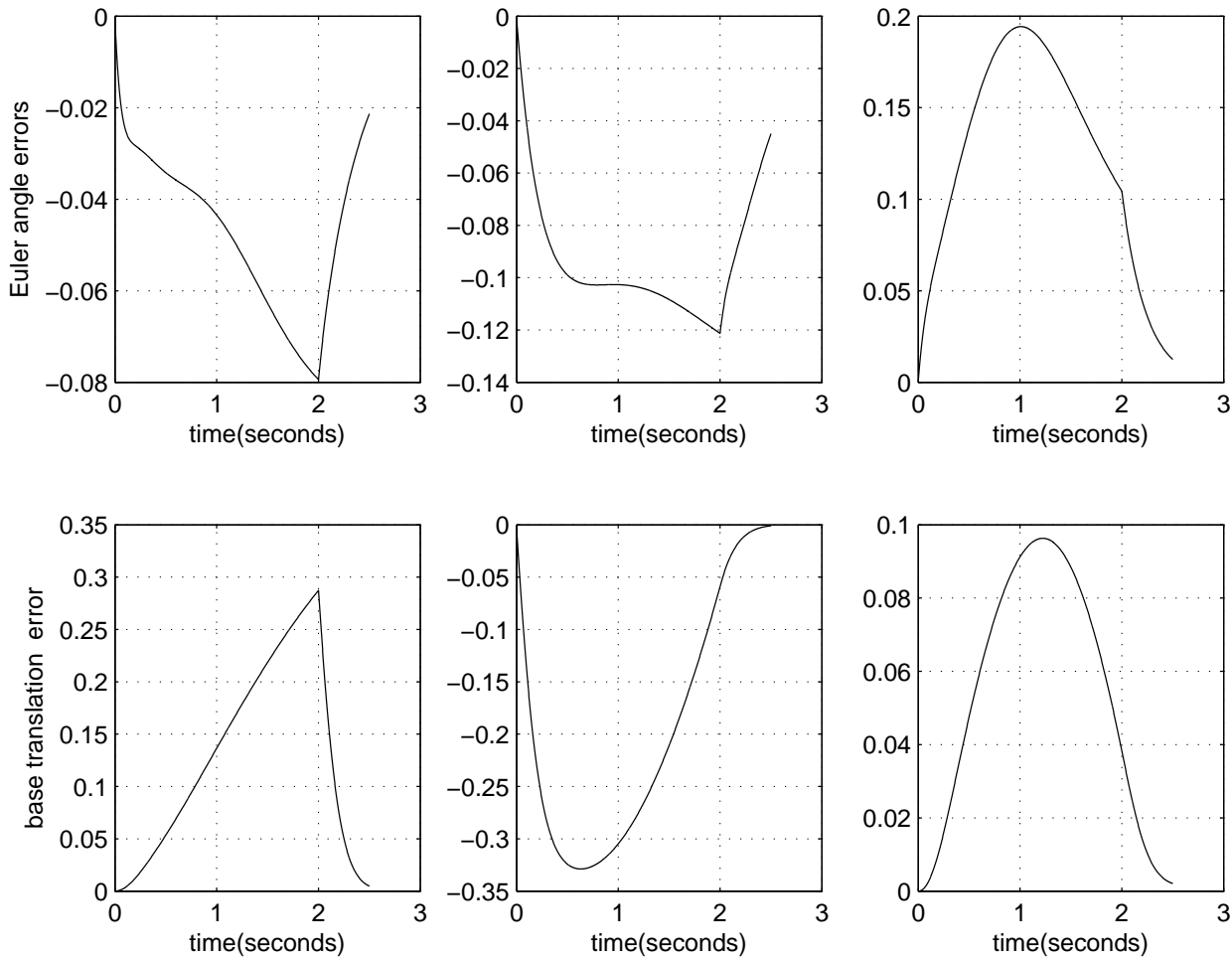

Figure 4. The control position errors (in radians and meters) as functions of time. These errors mean that the achieved coordination could be better. The system falls behind in time in following the reference input signals that define the desired coordination. Given enough time however, the system catches up with the inputs and all terminal errors tend to zero, as shown here. The system is stable. The point to point motion is adequate. Coordination, as specified by the inputs, is not adequate. 

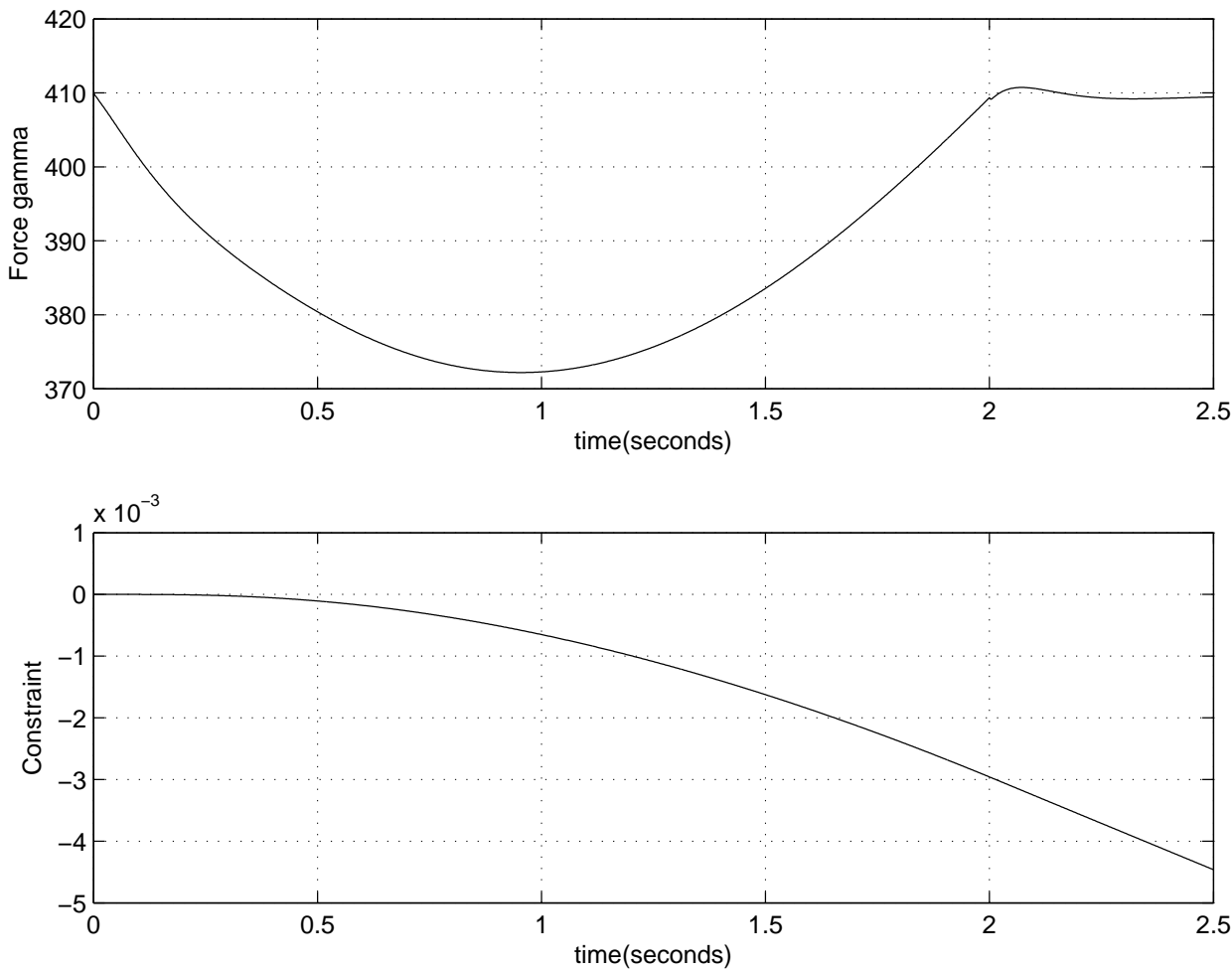

Figure 5. The force of contact in Newtons and the magnitude of the constraint in meters as functions of time.
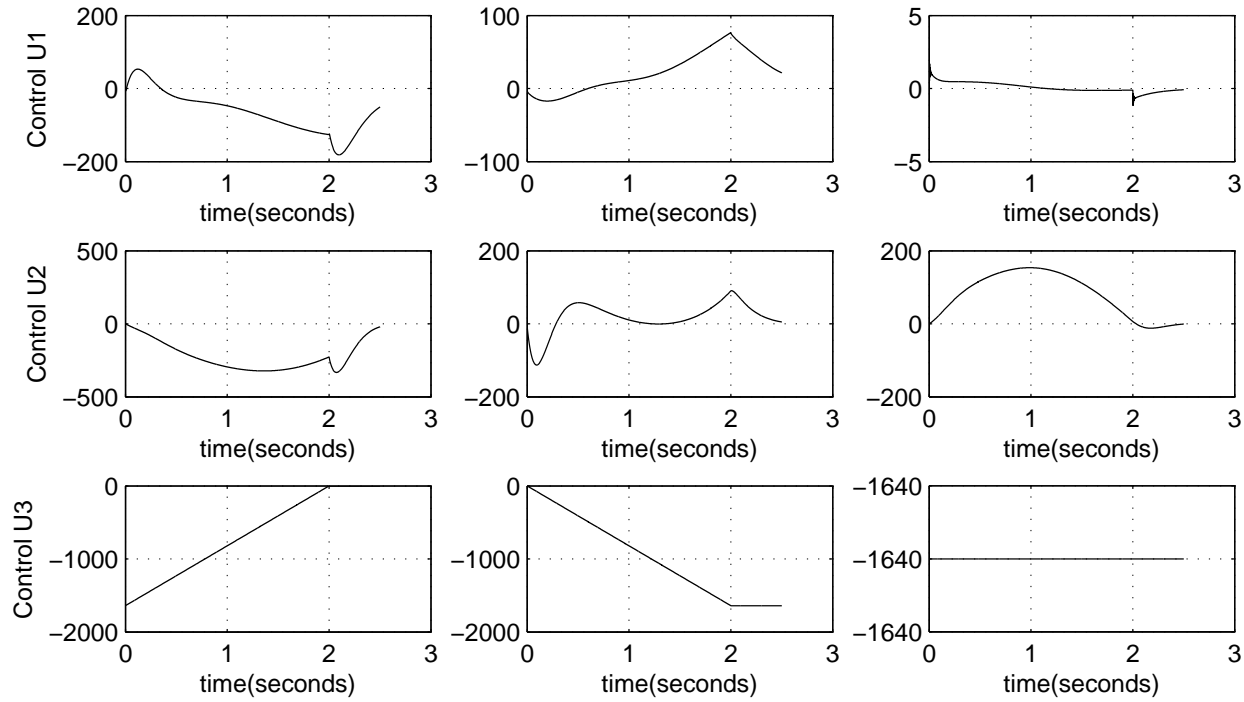

Figure 6. Control signals $U_{1}$, in Newton-meters, $U_{2}$ and $U_{3}$ in Newtons. as functions of time

\section{Acknowledgments}

The author would like to thank professor Joel Johnson, Chair of the Electrical and Computer Engineering Department at The Ohio State University for support and encouragement. The author is indebted to Robert J. Garbacz, Nelson Kent Brooks and Phil Peer for intellectual and technical support.

The author gratefully acknowledges the technical support of the Old Worthington Library and its staff and associates and in particular, Gena Smith. 


\section{References}

Basmajian, J. (1978). Muscles alive. The Williams and Wilkins Company.

Boileau Grant, R. (1962). An atlas of anatomy (fifth ed.). The Williams Wilkins Co.

Brooks, V. (1986). The neural basis of motor control. Oxford University Press.

Byrnes, C. I., \& Issidori, A. (1991). Asymptotic stabilization of minimum phase nonlinear systems. IEEE Trans. Automatic Control, 36, 1122-1137.

Craig, J. J. (1989). Introduction to robotics, mechanics and control. Prentive Hall.

Doeringer, J., \& Hogan, N. (1998, October-November). Serial processing in human movement production. Neural Networks, 11 (7 and 8), 1345,1356.

Evarts, E. (1975). Changing concepts of central control of movement. Can. J. Physiology, 53, 191 - 201.

Evarts, E., Bizzi, E., Burke, R., DeLong, M., \& Thach, W., Jr. (1971). Central control of movement. Neuroscience Res. Prog. Bulletin, 1 -170.

Hemami, H., \& Dariush, B. (2000). Single rigid body representation, control and stability for robotic applications. In Proc. irac 2000, san francisco, submitted. IEEE Robotic Society.

Hemami, H., \& Hemami, M. (2014). Modeling and monitoring of passive control structures in human movement. International J. of Control, 87, 1861-1876.

Hemami, H., \& Hemami, M. (2016). State space methods and examples for computational models of human movement. Mechanical Engineering Research, 6(1), 46-65.

Hemami, H., Tarr, E., Li, B., Krishnamurthy, A., Clymer, B., \& Darius, B. (2016). Towards a cybernetic model of human movement. Mechanical Engineering Research, 6(1), 1-18.

Hemami, H., \& Utkin, V. (2002, April). On the dynamics and lyapunov stability of constrained and embedded rigid bodies. International J. of Control, 75(6), 408-420.

Henneman, E. (1980). organization of the spinal cord and its reflexes. In V. Mountcastle (Ed.), Medical physiology (Vol. 1, p. 762-786). The C.V. Mosby Company.

Iqbal, K., \& Roy, A. (2009, January). A novel theoretical framework for the dynamic stability analysis, movement control, and trajectory generation in a multisegment biomechanical model. J. of Biomechnical Eng, Trans. ASME, 131(1), 002-013.

Ivanenko, Y., Grasso, R., Macellari, V., \& Lacquaniti, F. (2002). Spinal control of support and contact forces. Journal of Physiology, 87, 3070-3089.

Kandel, E., Schwarrtz, J., \& Jessell, T. (1991). Principles of neural science (Third ed.). Elsevir.

Kandel, R., E., \& Jessell, M., T. (1991). Touch. In Principles of neural science (Third ed., p. 367 - 384). New York: Elsevir.

Kapandji, I. A. (1979). The physiology of the joints, volume 2 (first ed.). Edinburgh: Churchill livingstone.

Kendall, O., Kendall, F. P., \& Wadsworth, G. E. (1971). Muscles testing and function (second ed.). The Williams Wilkins Co.

Kornhuber, H. H. (1971). Motor functions of cerebellum and basal ganglia: The cerebellocortical saccadic (ballistic) clock, the cerebellonuclear hold regulator, and the basal ganglia ramp (voluntary speed smooth movement) generator. Kybernetik, 8 Bd., Heft 4, 157-162.

Kornhuber, H. H. (1973). Cerebral corex, cerebellum, and basal ganglia: And introduction to their motor functions. The Neurosciences, III, 267-280.

Lacquaniti, F., \& Maioli, C. (1994). independent control of limb position and contact forces in cat posture. Journal of Neurophysiology, 72, 1476-1495. 
Nashner, L., \& McCollum, G. (1985). The organization of human postural movements: a formal basis and experimental synthesis. Behv.Brain Science, 8, 132-175.

Nicholls, J., Martin, A., \& Wallace, B. (1992). From neuron to brain.

Ozbay, H. (2000). Introduction tofeedback control theory. Boca Raton, Florida: CRC Press.

Pai, Y.-C., \& Iqbal, K. (1999). Simulated movement termination for balance recovery: can movement strategies be sought to maintain stability in the presence of slipping or forced sliding? J. Biomechanics., $779-786$.

Paul, R. P. (1982). Robot manipulators, mathematics,programming and control. The M.I.T. Press.

Prescott, A. S., \& Kononck, Y. D. (2005). Integration time in a subset of spinal lamina i neurons is lengthened by sodium and calcium currents acting synergistically to prolong sub threshold depolarization. J. Neuroscience, 4743 - 4754.

Quental, C., Folgado, J., Ambrocio, J., \& Monteiro, J. (2012). A multibody biomechanical model of the upper limb including the shoulder girdle. Multibody Systems Dynamics, 83-108.

Rosse, C., \& Clawson, D. K. (Eds.). (1970). Introduction to the musculoskeletal system (first ed.). Harper and Row.

Shabana, A. (1985). Viscoelastic analysis of multibody systems using the finite element method. J. of Sound and Vibration, 271-284.

Szturm, T., \& Fallang, B. (1998). Effects of varying acceleration of platform translation and toes-up rotations on the pattern and magnitude of balance reactions in humans. Journal of Vestibular Research, 8(5), 381-397.

Tomovic, R., Popovic, D., \& Stein, R. B. (Eds.). (1995). Nonanalytical methods for motor control (first ed.). World Scientific.

W., K. S., \& Nicholls, J. (n.d.). From neuron to brain a cellular approach to the function of the nervous system.

Waldron, K., \& Schmiedeler, J. (2008). Kinematics. In Handbook of robotic systems, 1161 pages. Springer, New York.

Warfel, J. H. (1978). The extremities (Fourth ed.). Philadelphia: Lea and Febiger.

Winter, D., Patla, A., Prince, F., Ishac, M., \& K.Gielo-Perczak. (1998). Stiffness control of balance in quiet standing. J. Neurophysiol, 80, 1211-1221.

\section{Appendix A}

The matrices that appear in the dynamics of the system are:

Let $A_{1}\left(\theta_{1}\right), A_{2}\left(\theta_{2}\right)$ and $A_{3}\left(\theta_{3}\right)$ be defined by

$$
\begin{aligned}
& A_{1}\left(\theta_{1}\right)=\left[\begin{array}{ccc}
1 & 0 & 0 \\
0 & \cos \theta_{1} & -\sin \theta_{1} \\
0 & \sin \theta_{1} & \cos \theta_{1}
\end{array}\right] . \\
& A_{2}\left(\theta_{2}\right)=\left[\begin{array}{ccc}
\cos \theta_{2} & 0 & \sin \theta_{2} \\
0 & 1 & 0 \\
-\sin \theta_{2} & 0 & \cos \theta_{2}
\end{array}\right] . \\
& A_{3}\left(\theta_{3}\right)=\left[\begin{array}{ccc}
\cos \theta_{3} & -\sin \theta_{3} & 0 \\
\sin \theta_{3} & \cos \theta_{3} & 0 \\
0 & 0 & 1
\end{array}\right] .
\end{aligned}
$$

Now $A(\Theta)$ can be defined:

$$
A(\Theta)=A_{1}\left(\theta_{1}\right) A_{2}\left(\theta_{2}\right) A_{3}\left(\theta_{3}\right)
$$


Table 1: Definition of symbols and numerical values for the wiper.

\begin{tabular}{lccc}
\hline & symbol & value & unit \\
\hline mass & $\mathrm{m}$ & 41.00 & $\mathrm{Kg}$ \\
p.m. of i. & $\mathrm{j} 1$ & 2.00 & $\mathrm{Kg} \mathrm{m}^{2}$ \\
p.m. of i. & $\mathrm{j} 2$ & 2.00 & $\mathrm{Kg} \mathrm{m}^{2}$ \\
p.m. of i. & $\mathrm{j} 3$ & 0.40 & $\mathrm{Kg} \mathrm{m}$ \\
c. of g. & 1 & 0.42 & $\mathrm{~m}$ \\
gravity & $\mathrm{g}$ & 0.00 & $\mathrm{~m} / \mathrm{s}^{2}$ \\
\hline
\end{tabular}

For reference purposes, matrix $A$ is reproduced here in detail ( see also Craig (1989).)

$$
A=\left[\begin{array}{ccc}
\cos \theta_{3} \cos \theta_{2} & -\sin \theta_{3} \cos \theta_{2} & \sin \theta_{2} \\
\cos \theta_{1} \sin \theta_{3}+\sin \theta_{1} \sin \theta_{2} \cos \theta_{3} & \cos \theta_{1} \cos \theta_{3}-\sin \theta_{1} \sin \theta_{2} \sin \theta_{3} & \sin \theta_{1} \cos \theta_{2} \\
\sin \theta_{1} \sin \theta_{3}-\cos \theta_{1} \sin \theta_{2} \cos \theta_{3} & \sin \theta_{1} \cos \theta_{3}+\cos \theta_{1} \sin \theta_{2} \sin \theta_{3} & \cos \theta_{1} \cos \theta_{2}
\end{array}\right] .
$$

The matrix $B(\Theta)$ is given by:

$$
B(\Theta)=\left[\begin{array}{ccc}
\frac{\cos \theta_{3}}{\cos \theta_{2}} & \frac{-\sin \theta_{3}}{\cos \theta_{2}} & 0 \\
\sin \theta_{3} & \cos \theta_{3} & 0 \\
\frac{-\sin \theta_{2} \cos \theta_{3}}{\cos \theta_{2}} & \frac{\sin \theta_{2} \sin \theta_{3}}{\cos \theta_{2}} & 1
\end{array}\right] .
$$

Let vector $R$ have components $r_{1}, r_{2}$, and $r_{3}$. The skew symmetric matrix $\breve{\mathcal{R}}$ is defined as:

$$
\begin{gathered}
\breve{\mathcal{R}}=\left[\begin{array}{ccc}
0 & -r_{3} & r_{2} \\
r_{3} & 0 & -r_{1} \\
-r_{2} & r_{1} & 0
\end{array}\right] . \\
f=\breve{\Omega} J(\Omega)
\end{gathered}
$$

The numerical values for the physical parameters of the wiper in the simulations Hemami \& Dariush (2000) are given in Table 1.

The remaining wiper parameters for the simulation are

$R=[0,0,-0.42]^{\prime}$

$K R_{a}=\operatorname{Diag}(3000,3000,1500)$

$L R_{a}=\operatorname{Diag}(750,750,375)$

$K T_{a}=\operatorname{Diag}(3000,3000,1500)$

$L T_{a}=\operatorname{Diag}(750,750,375)$

$K_{f}=50$

\section{Copyrights}

Copyright for this article is retained by the author(s), with first publication rights granted to the journal.

This is an open-access article distributed under the terms and conditions of the Creative Commons Attribution license (http://creativecommons.org/licenses/by/4.0/). 